\title{
Lung volumes predict survival in patients with chronic lung allograft dysfunction
}

\author{
Nikolaus Kneidinger ${ }^{1,8}$, Katrin Milger ${ }^{1,8}$, Silke Janitza², Felix Ceelen ${ }^{1}$, \\ Gabriela Leuschner ${ }^{1}$, Julien Dinkel ${ }^{3}$, Melanie Königshoff $\mathbb{C}^{4}$, Thomas Weig ${ }^{5}$, \\ René Schramm ${ }^{6}$, Hauke Winter ${ }^{7}$, Jürgen Behr ${ }^{1}$ and Claus Neurohr ${ }^{1}$
}

Affiliations: ${ }^{1}$ Dept of Internal Medicine V, Comprehensive Pneumology Center (CPC-M), Member of the German Center for Lung Research (DZL), University of Munich, Munich, Germany. ${ }^{2}$ Dept of Medical Informatics, Biometry and Epidemiology, LMU Munich, Munich, Germany. ${ }^{3}$ Institute for Clinical Radiology, Comprehensive Pneumology Center (CPC-M), Member of the German Center for Lung Research (DZL), University of Munich, Munich, Germany. ${ }^{4}$ Comprehensive Pneumology Center (CPC-M), Research Unit Lung Repair and Regeneration, Helmholtz Zentrum München, Member of the German Center for Lung Research (DZL), Munich, Germany. ${ }^{5}$ Dept of Anaesthesiology, University of Munich, Munich, Germany. ${ }^{6} \mathrm{Clinic}$ of Cardiac Surgery, University of Munich, Munich, Germany. ${ }^{7}$ Dept of Thoracic Surgery, University of Munich, Munich, Germany. ${ }^{8}$ Both authors contributed equally.

Correspondence: Nikolaus Kneidinger, Department of Internal Medicine V, University of Munich, Comprehensive Pneumology Center (CPC-M), Member of the German Center for Lung Research (DZL), Marchioninistrasse 15, 81377 Munich, Germany. E-mail: nikolaus.kneidingeramed.uni-muenchen.de

@ERSpublications

Measurement of lung volumes in lung transplant recipients allows identification of patients at risk of poor outcome http://ow.ly/FCzy3084Al2

Cite this article as: Kneidinger N, Milger K, Janitza S, et al. Lung volumes predict survival in patients with chronic lung allograft dysfunction. Eur Respir J 2017; 49: 1601315 [https://doi.org/10.1183/13993003.013152016].

ABSTRACT Identification of disease phenotypes might improve the understanding of patients with chronic lung allograft dysfunction (CLAD). The aim of the study was to assess the impact of pulmonary restriction and air trapping by lung volume measurements at the onset of CLAD.

A total of 396 bilateral lung transplant recipients were analysed. At onset, CLAD was further categorised based on plethysmography. A restrictive CLAD (R-CLAD) was defined as a loss of total lung capacity from baseline. CLAD with air trapping (AT-CLAD) was defined as an increased ratio of residual volume to total lung capacity. Outcome was survival after CLAD onset. Patients with insufficient clinical information were excluded ( $\mathrm{n}=95)$.

Of 301 lung transplant recipients, 94 (31.2\%) developed CLAD. Patients with R-CLAD ( $\mathrm{n}=20)$ and AT-CLAD $(n=21)$, respectively, had a significantly worse survival $(\mathrm{p}<0.001)$ than patients with non-R/ AT-CLAD. Both R-CLAD and AT-CLAD were associated with increased mortality when controlling for multiple confounding variables (hazard ratio (HR) 3.57, 95\% CI 1.39-9.18; $\mathrm{p}=0.008$; and HR 2.65, 95\% CI 1.05-6.68; $\mathrm{p}=0.039$ ). Furthermore, measurement of lung volumes was useful to identify patients with combined phenotypes.

Measurement of lung volumes in the long-term follow-up of lung transplant recipients allows the identification of patients who are at risk for worse outcome and warrant special consideration.

This article has supplementary material available from erj.ersjournals.com

Received: July 042016 | Accepted after revision: Dec 152016

Support statement: This work was supported by the German Center for Lung Research (DZL). Funding information for this article has been deposited with the Crossref Funder Registry.

Conflict of interest: None declared.

Copyright OERS 2017 


\section{Introduction}

Chronic lung allograft dysfunction (CLAD) is the main limiting factor for long-term survival after lung transplantation. It is defined as a decline in the persistent forced expiratory volume in $1 \mathrm{~s}$ (FEV1) of $>20 \%$ from the best value achieved, in the absence of other causes [1]. However, CLAD has a heterogeneous nature, which has been recognised as one of the major challenges in transplant medicine. Differentiating the phenotypes of CLAD may be a further step to precision medicine in lung transplant recipients, potentially helping to identify high-risk patients.

Recently, a restrictive form of CLAD has been reported $[2,3]$. This condition, called restrictive allograft syndrome (RAS), is associated with parenchymal and pleural thickening, and characterised by a more progressive course of disease. Diagnostic criteria are based on pulmonary function tests, imaging studies and histopathological characteristics in the context of the clinical course [3].

However, the ongoing debate about the diagnostic approach to CLAD, its terminology and its syndromes illustrates the complexity of this condition $[1,4,5]$. In some instances a clear discrimination might not be possible, because RAS and bronchiolitis obliterans syndrome (BOS) might occur consecutively or even simultaneously [3].

Given that the optimal diagnostic approach and its prognostic value remain uncertain, the identification of physiological traits might permit the phenotyping of patients with CLAD and proactive identification of patients at risk of poor outcomes. In this respect, post-transplant analysis of spirometric indices and the identification of specific lung function patterns have been proposed [4]. In particular, two recent studies demonstrated that a forced vital capacity (FVC) decline at CLAD onset, suggestive of a restrictive ventilatory defect, was associated with poor outcome $[6,7]$.

In addition to spirometric indices, the measurement of static lung volumes and lung capacities might be useful to identify and differentiate particular physiological phenotypes. In this context, SATO et al. [3] demonstrated that a decline of $10 \%$ in total lung capacity (TLC) had a high diagnostic accuracy for RAS, with low TLC associated with poor outcome [8]. Air trapping can also be assessed by lung volume measurements, and, in patients with obstructive lung diseases, may be more important than parameters of airflow in terms of functional limitation, dyspnoea and outcome [9-11]. However, the impact of air trapping in patients with CLAD remains unknown.

The value of routine measurements of static lung volumes and lung capacities, in addition to spirometry, has not been studied so far. The aim of this study was thus to assess the diagnostic and prognostic impact of lung volume measurements in patients with CLAD.

\section{Methods}

Patients and study design

All patients who underwent bilateral lung transplantation at our centre from April 1990 to December 2013 $(n=396)$ were included in the study and analysed retrospectively. The study was conducted at the University of Munich, Germany, and approved by the local ethics committee (UE no. 630-15).

Pre-transplant diagnoses were categorised into interstitial lung disease (ILD), chronic obstructive pulmonary disease (COPD), cystic fibrosis and "others" [12].

Patients were followed until death, retransplantation or March 31, 2015. Outcome was survival after CLAD onset (time to death or retransplantation), as described previously $[6,8]$.

Assessment and surveillance of lung transplant recipients, as well as categorisation of the causes of death, are described in the supplementary material.

\section{Chronic lung allograft dysfunction}

CLAD was defined as a persistent decline in FEV1 by $20 \%$ from baseline FEV1 (FEV1/FEV $1_{\text {baseline }}<80 \%$ ) that was present for a minimum of 3 weeks according to the current recommendations [13, 14]. Baseline FEV1 ( $\left.F E V 1_{\text {baseline }}\right)$ was defined as the mean of the two best measurements of FEV1 post-transplant, at least 3 weeks apart. Patients with CLAD potentially caused by confounding conditions were excluded from the analysis $(\mathrm{n}=8)$ (figure 1$)$.

Baseline values of FVC ( $\left.\mathrm{FVC}_{\text {baseline }}\right)$, residual volume $\left(\mathrm{RV}_{\text {baseline }}\right)$ and TLC ( $\left.\mathrm{TLC}_{\text {baseline }}\right)$ were defined as the mean of the two measures at the time of the two best FEV1 values.

The ratio of FEV1 to FVC (FEV1/FVC) and the ratio of RV to TLC (RV/TLC) were assessed from the single measurement at the time of CLAD onset. Additionally, changes over time were expressed as a ratio of the measurement at CLAD onset to the mean of the two measures at the time of the two best FEV1 values (FEV1/FVC baseline (\%) and $\mathrm{RV} / \mathrm{TLC}_{\text {baseline }}(\%)$, respectively). 


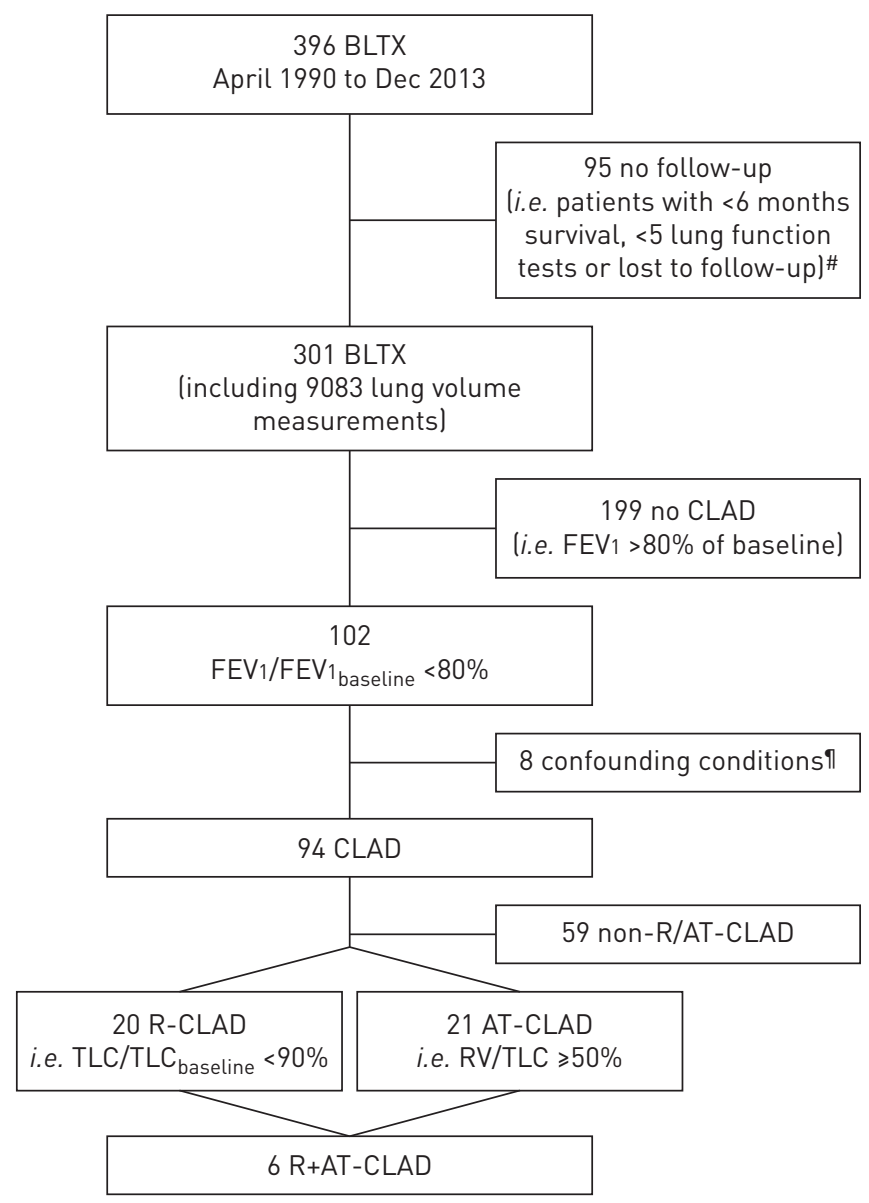

FIGURE 1 Description of the study cohort and classification according to lung capacity/volume measurements. BLTX: bilateral lung transplantation; CLAD: chronic lung allograft dysfunction; FEV1: forced expiratory volume in $1 \mathrm{~s}$; RV: residual volume; TLC: total lung capacity; R-CLAD: restrictive CLAD; AT-CLAD: CLAD with air trapping; R+AT-CLAD: both R- and AT-CLAD; non-R/AT-CLAD: neither R- nor AT-CLAD. " : <6 months survival, $n=65 ;<5$ lung function tests, $n=24$; lost to follow-up, $n=6$. ": malignancy coincident with lung function decline, $\mathrm{n}=4$; significant large airway disease, $\mathrm{n}=2$; uncontrolled infection, $\mathrm{n}=2$. In total, 94 patients developed CLAD, 59 non-R/AT-CLAD, 20 R-CLAD, 21 AT-CLAD, of which 6 developed both R- and AT-CLAD.

Restrictive CLAD (R-CLAD) was defined as a persistent drop in TLC of $>10 \%$ of baseline (i.e. TLC/ TLC $_{\text {baseline }}<90 \%$ ) at the time of CLAD onset, as reported previously [3]. Air trapping at CLAD onset (AT-CLAD) was defined as an increase in the ratio of RV to TLC of $\geqslant 50 \%$ (i.e. RV/TLC $\geqslant 50 \%$ ).

The clinical syndrome RAS was defined as a restrictive lung function pattern $\left(\mathrm{FVC} / \mathrm{FVC}_{\mathrm{baseline}}<80 \%\right.$, FEV1/FVC $>70 \%$ or TLC/TLC baseline $<90 \%$ ) at the time of CLAD onset $[3,6]$ and the presence of persistent parenchymal infiltrates and/or (sub)pleural thickening on imaging studies $[3,4]$ in the context of the lung function decline [15]. All patients with CLAD were retrospectively assessed for the predominant clinical syndrome by two independent transplant physicians.

Patients with neither R-CLAD nor AT-CLAD were classified as non-R/AT-CLAD and served as the control group.

Time to CLAD was defined as the time from transplantation to the date of the first of the two measurements used to confirm CLAD. Severity of CLAD at onset was assessed by the absolute value of FEV1/FEV $1_{\text {baseline. }}$ Severe-onset CLAD and early-onset CLAD were defined as $\mathrm{FEV} 1 / \mathrm{FEV} 1_{\text {baseline }} \leqslant 65 \%$ at CLAD onset and onset of CLAD within 2 years of transplantation, respectively [16].

\section{Statistics}

A complete description of the statistics is provided in the supplementary material.

The Mann-Whitney U-test was used to test if two independent groups differed with respect to a metric variable. Differences between the groups with respect to categorical variables were tested using Fisher's exact test. 
The differences between the survival curves of two groups were tested using the log-rank test when hazards were proportional or nearly proportional [17], and the results were presented as Kaplan-Meier curves [18].

Because the present data fulfilled the assumption of proportional hazards, we used Cox regression to model survival times in dependence of multiple risk factors [19]. To allow for the modelling of smooth nonlinear effects, we used penalised splines implemented in the function spline of the $\mathrm{R}$ package "survival". Wald tests were used to assess the significance of the risk factors in the Cox models.

A significance level of $5 \%$ was used for all statistical tests. All analyses were performed using the statistical software R, version 3.0.1 [20].

\section{Results}

\section{Study population}

In total, 396 patients underwent bilateral lung transplant over the study period. Of these, 95 patients with insufficient clinical information were excluded. The final study population consisted of 301 patients. Enrolment is depicted in figure 1.

Mean \pm SD age at the time of transplantation was $42.3 \pm 12.6$ years. A total of 164 patients (54.5\%) were female. Mean \pm SD follow-up was $5.84 \pm 4.36$ years. During the study period, 9083 lung function measures were assessed, with a mean \pm SD of $30.9 \pm 19.9$ lung function measures per patient.

\section{Chronic lung allograft dysfunction}

A total of 102 patients (33.9\%) developed FEV1 decline that fulfilled the definition of CLAD. However, in eight of these patients $(7.8 \%)$, confounding conditions were present and they were excluded from further analysis (figure 1). In 53 of the remaining 94 patients with CLAD (31.2\%), the combined endpoint death $(\mathrm{n}=48 ; 90.6 \%)$ or retransplantation $(\mathrm{n}=5 ; 9.4 \%)$ was observed.

Median overall survival of the 94 patients with CLAD was 7.30 years, and median survival upon CLAD onset was 2.36 years. Time to CLAD was $4.08 \pm 3.73$ years.

\section{Restrictive CLAD}

Of the 94 patients with CLAD, 20 patients (21.3\%) with a concomitant loss of TLC (i.e. TLC/TLC baseline $<90 \%$ ) at CLAD onset were classified as R-CLAD (figure 1). As shown in table 1, patients with R-CLAD were more likely to have COPD (40.0\% versus $10.2 \%$; $=0.005)$ and less likely to have ILD ( $15.0 \%$ versus $44.1 \% ; \mathrm{p}=0.030)$ as the pre-transplant diagnosis than patients with CLAD who had neither R- nor AT-CLAD (non-R/AT-CLAD). CLAD occurred earlier in patients with R-CLAD (2.6 \pm 2.5 years versus $5.0 \pm 4.2$ years; $\mathrm{p}=0.005)$. In total, 11 patients $(55.0 \%)$ had a concomitant loss of $\mathrm{FVC} / \mathrm{FVC}_{\text {baseline }}<80 \%$ (table 1 ).

Survival time upon CLAD onset was significantly lower in patients with R-CLAD than in patients with non-R/ AT-CLAD (log-rank test, $\mathrm{p}<0.0001$ ); 2-year survival rates upon CLAD onset were $28.6 \%$ (95\% CI 14.0-58.3\%) versus $76.1 \%$ (95\% CI 65.0-89.1\%) and median survival times were 303 days versus 2689 days (figure 2a).

In the multiple Cox regression, R-CLAD was significantly associated with worse outcome (hazard ratio (HR) 3.57, 95\% CI 1.39-9.18; $\mathrm{p}=0.008$ ) when controlling for age, sex, pre-transplant diagnosis, time to CLAD, severity of CLAD at onset, approach of thoracotomy, type of immunosuppression, use of azithromycin and year of transplantation (table 2).

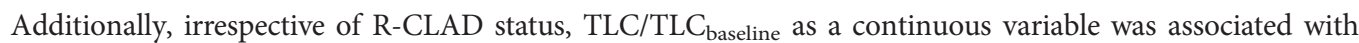

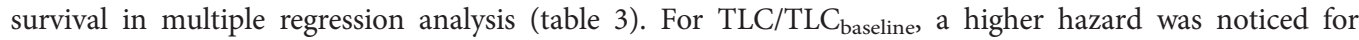

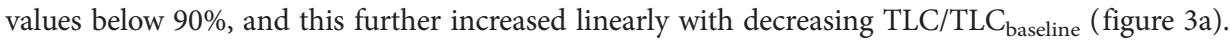

Patients who developed R-CLAD within 2 years of transplantation had a tendency for worse survival than patients who developed R-CLAD beyond 2 years after transplantation (log-rank test, $\mathrm{p}=0.074$; supplementary figure $\mathrm{S} 1$ ).

In total, 17 patients (85.0\%) with R-CLAD died during the study period. Cause of death was CLAD-related in 14 of the 17 patients (82.4\%). In nine of these patients $(64.3 \%)$, cause of death was infection-associated, as described in supplementary table S1.

Radiographic findings on chest computed tomography (CT) scan at the time of R-CLAD onset are shown in table 4 .

18 (90\%) patients with R-CLAD received the final clinical diagnosis of RAS, compared with only one patient in the non-R-CLAD group $(\mathrm{p}<0.0001)$. R-CLAD was therefore associated with a high diagnostic accuracy for RAS (sensitivity 95\%, specificity $97 \%$, positive predictive value $90 \%$, negative predictive value $99 \%$ ). 
TABLE 1 Characteristics of patients with chronic lung allograft dysfunction (CLAD) according to the lung function pattern at CLAD onset

\begin{tabular}{|c|c|c|c|c|}
\hline & CLAD (all) & Non-R/AT-CLAD & R-CLAD & AT-CLAD \\
\hline Subjects & 94 (100) & $59(62.8)$ & 20 (21.3) & $21(22.3)$ \\
\hline Age years & $41.2 \pm 12.9$ & $39.6 \pm 13.0$ & $44.1 \pm 13.6$ & $46.3 \pm 11.2^{*}$ \\
\hline Females & 52 (55.3) & $34(57.6)$ & $10(50.0)$ & $12(57.1)$ \\
\hline \multicolumn{5}{|l|}{ Disease } \\
\hline ILD & 35 (37.2) & $26(44.1)$ & $3(15.0)^{*}$ & 7 (33.3) \\
\hline COPD & $16(17.0)$ & $6(10.2)$ & $8(40.0)^{* *}$ & $6(28.6)$ \\
\hline Cystic fibrosis & $27(28.7)$ & 18 (30.5) & $4(20.0)$ & $5(23.8)$ \\
\hline Others & $16(17.0)$ & $9(15.3)$ & $5(25.0)$ & $3(14.3)$ \\
\hline SO-CLAD & $33(35.1)$ & $15(25.4)$ & $10(50.0)$ & $11(52.4)^{*}$ \\
\hline EO-CLAD & $22(23.4)$ & 9 (15.3) & $5(25.0)$ & $9(42.9)^{*}$ \\
\hline Time to CLAD years & $4.1 \pm 3.7$ & $5.0 \pm 4.2$ & $2.6 \pm 2.5^{* *}$ & $2.3 \pm 1.5^{* *}$ \\
\hline FEV1/FEV1 $1_{\text {baseline }} \%$ & $62.2 \pm 12.6$ & $72.0 \pm 10.4$ & $68.3 \pm 14.9$ & $62.0 \pm 14.5^{* * *}$ \\
\hline FVC/FVC baseline $\%$ & $87.9 \pm 15.4$ & $94.0 \pm 11.8$ & $75.2 \pm 17.8^{* * *}$ & $78.1 \pm 14.9 * * *$ \\
\hline FEV1/FVC $\%$ & $67.9 \pm 14.8$ & $64.7 \pm 11.8$ & $82.3 \pm 11.7^{* * *}$ & $67.4 \pm 18.5$ \\
\hline FEV1/FVC baseline $\%$ & $81.1 \pm 13.7$ & $77.4 \pm 11.1$ & $94.6 \pm 11.1^{* * *}$ & $84.6 \pm 18.2^{*}$ \\
\hline $\mathbf{R V} / \mathbf{R V}_{\text {baseline }} \%$ & $116.5 \pm 39.7$ & $120.3 \pm 41.9$ & $92.6 \pm 30.6 * *$ & $127.1 \pm 30.0$ \\
\hline TLC/TLC baseline $\%$ & $97.0 \pm 13.0$ & $101.3 \pm 9.8$ & $80.4 \pm 10.8^{* * *}$ & $96.5 \pm 12.1$ \\
\hline RV/TLC \% & $43.8 \pm 9.1$ & $40.1 \pm 5.6$ & $44.6 \pm 9.4 *$ & $56.8 \pm 6.2^{* * *}$ \\
\hline RV/TLC baseline \% & $118.0 \pm 30.5$ & $115.7 \pm 27.7$ & $116.4 \pm 40.0$ & $132.2 \pm 38.5^{*}$ \\
\hline FEV1/FVC $<70 \%$ & $54(57.4)$ & $39(66.1)$ & $3(15.0)^{* * *}$ & 13 (61.9) \\
\hline $\mathrm{FVC} / \mathrm{FVC}_{\text {baseline }}<90 \%$ & $49(52.1)$ & 19 (32.2) & $17(85.0)^{* * *}$ & $18(85.7)^{* * *}$ \\
\hline $\mathrm{FVC} / \mathrm{FVC}_{\text {baseline }}<80 \%$ & $23(24.5)$ & 5 (8.5) & $11(55.0)^{* * *}$ & $11(52.4)^{* * *}$ \\
\hline
\end{tabular}

Data are presented as mean \pm SD or $n(\%)$. R-CLAD: restrictive CLAD; AT-CLAD: CLAD with air trapping; non-R/ AT-CLAD: neither R-CLAD nor AT-CLAD; ILD: interstitial lung disease; COPD: chronic obstructive pulmonary disease; SO-CLAD: severe-onset CLAD (decline in FEV1 to $\leqslant 65 \%$ of FEV1 baseline $_{\text {at }}$ CLAD onset); EO-CLAD: early-onset CLAD (onset of CLAD within 2 years of transplantation); FEV1: forced expiratory volume in $1 \mathrm{~s}$; FVC: forced vital capacity; RV: residual volume; TLC: total lung capacity. Non-R/AT-CLAD was compared with R-CLAD and AT-CLAD, respectively. ${ }^{*}: p<0.05 ;{ }^{* *}: p<0.01 ;{ }^{* *} p<0.001$ (bold type indicates significant values).

\section{CLAD with air trapping}

21 patients $(22.3 \%$ of the 94 patients with CLAD) with a concomitant increase of RV/TLC $\geqslant 50 \%$ at CLAD onset were classified as AT-CLAD. As shown in table 1, patients with AT-CLAD were older than patients with non-R/AT-CLAD (46.3 \pm 11.2 years versus $39.6 \pm 13.0$ years; $\mathrm{p}=0.039)$. Compared with those with non-R/AT-CLAD, CLAD occurred earlier (2.3 \pm 1.5 years versus $5.0 \pm 4.2$ years; $\mathrm{p}=0.004)$ and was more severe at onset $\left(\mathrm{FEV} 1 / \mathrm{FEV} 1_{\text {baseline }} 62.0 \pm 14.5 \%\right.$ versus $\left.72.0 \pm 10.4 \% ; \mathrm{p}=0.0003\right)$ in patients with AT-CLAD (table 1). In total, 13 patients $(61.9 \%)$ were obstructed (i.e. FEV1/FVC $<70 \%) .10$ patients with AT-CLAD (47.6\%) had a concomitant loss of $\mathrm{FVC/FVC}$ baseline $<80 \%$.

AT-CLAD was significantly associated with decreased survival (log-rank test, $\mathrm{p}<0.0001)$. 2-year survival estimation upon CLAD onset was 20.0\% (95\% CI 8.3-48.1\%) versus 76.1\% (95\% CI 65.0-89.1\%). Median survival times were 240 days versus 2689 days (figure 2b).

In multiple Cox regression analysis, AT-CLAD was associated with worse outcome (HR 2.65, 95\% CI 1.05-6.68; $\mathrm{p}=0.039$ ) when controlling for age, sex, pre-transplant diagnosis, time to CLAD, severity of CLAD at onset, approach of thoracotomy, type of immunosuppression, use of azithromycin and year of transplantation (table 2). Additionally, irrespective of AT-CLAD status, RV/TLC as a continuous variable was associated with survival in the multiple regression analysis (table 3). An increased mortality risk was observed for RV/TLC at $\sim 45 \%$, increasing with increasing RV/TLC (figure $3 \mathrm{~b}$ ).

Patients who developed AT-CLAD within 2 years of transplantation had a similar survival to patients who developed AT-CLAD beyond 2 years after transplantation (log-rank test, $\mathrm{p}=0.209$; supplementary figure S2).

In total, 17 patients $(81.0 \%)$ with AT-CLAD died within the study period. Cause of death was CLAD-related in 15 of 17 patients $(88.2 \%)$. Of these, cause of death was infection-associated in eight patients $(53.3 \%)$, as shown in supplementary table S1.

Radiographic findings on chest CT scan at the time of AT-CLAD onset are shown in table 4. 

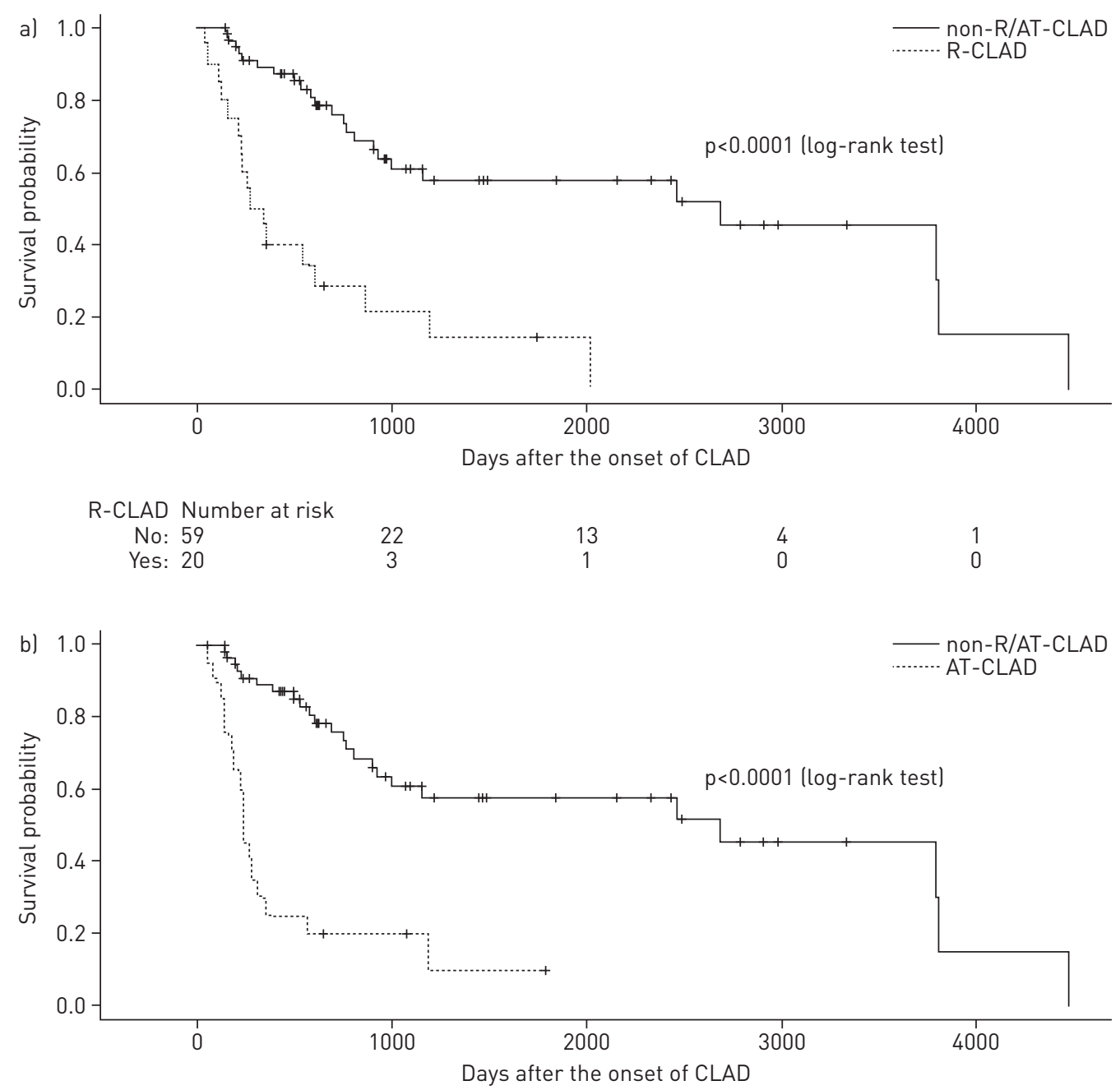

$\begin{array}{ccccc}\text { AT-CLAD Number at risk } & & & & \\ \text { No: } 59 & 22 & 13 & 4 & 1 \\ \text { Yes: } 21 & 3 & 0 & 0 & 0\end{array}$

FIGURE 2 Kaplan-Meier curves for patients with R-CLAD (total lung capacity (TLC)/TLC baseline $<90 \%$ ) and patients without R/AT-CLAD (a) and patients with AT-CLAD (residual volume/TLC $\geqslant 50 \%$ ) and patients without R/AT-CLAD (b). CLAD: chronic lung allograft dysfunction; AT-CLAD: CLAD with air trapping; R-CLAD: restrictive CLAD.

Longitudinal changes of RV/TLC, i.e. RV/TLC baseline (in \%), at CLAD onset did not add significant prognostic or diagnostic information. In the multiple Cox regression, RV/TLC seemed to have a better

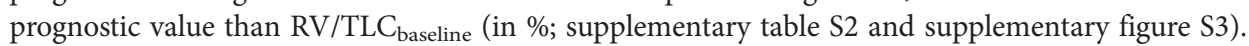

Non-R/AT-CLAD

A detailed description of patients with neither R- nor AT-CLAD is provided in table 1 and the supplementary material.

\section{$R-$ and $A T-C L A D(R+A T-C L A D)$}

Six (6.4\%) of the 94 patients with CLAD fulfilled the criteria for both R- and AT-CLAD, indicative of a

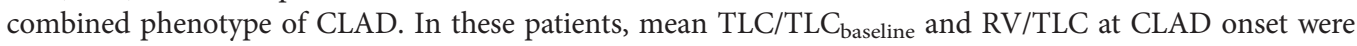
$83.3 \pm 8.4 \%$ and $55.0 \pm 6.2 \%$, respectively. A detailed presentation of the lung function values of patients with a combined phenotype is provided in the supplementary material (supplementary table S3). Survival time upon CLAD onset was significantly lower in patients with a combined phenotype than in patients with non-R/AT-CLAD (log-rank test, $\mathrm{p}=0.0013$ ). Median survival times were 308 days versus 2689 days. However, the survival curves did not differ between patients with a combined phenotype compared to 
TABLE 2 Multiple Cox regression models assessing the effect of restrictive chronic lung allograft dysfunction (R-CLAD) and chronic lung allograft dysfunction with air trapping (AT-CLAD) on survival

\begin{tabular}{llc} 
Covariate & HR (95\% Cl) & p-value \\
\hline R-CLAD (ref.: non-R/AT-CLAD) & $3.57(1.39-9.18)$ & $\mathbf{0 . 0 0 8}$ \\
Age & $0.98(0.94-1.01)$ & 0.177 \\
Sex (ref.: male) & $0.43(0.20-0.91)$ & $\mathbf{0 . 0 2 8}$ \\
Underlying disease (ref.: CF) & & \\
$\quad$ COPD & $1.23(0.41-3.72)$ & 0.708 \\
ILD & $1.35(0.37-4.90)$ & 0.647 \\
$\quad$ Others & $1.45(0.37-5.67)$ & 0.592 \\
Severity of CLAD, FEV1/FEV1 ${ }_{\text {baseline } \%}$ & $0.98(0.96-1.00)$ & 0.093 \\
Time to CLAD years & $0.93(0.79-1.10)$ & 0.414 \\
Anterolateral thoracotomy (ref.: clamshell thoracotomy) & $1.44(0.51-4.00)$ & 0.490 \\
Azithromycin (ref.: no azithromycin) & $0.22(0.07-0.71)$ & $\mathbf{0 . 0 1 1}$ \\
Cyclosporine A (ref.: tacrolimus) & $1.08(0.38-3.13)$ & 0.882 \\
Year of transplantation & $1.06(0.96-1.18)$ & 0.246 \\
AT-CLAD (ref.: non-R/AT-CLAD) & $2.65(1.05-6.68)$ & $\mathbf{0 . 0 3 9}$ \\
Age & $0.98(0.94-1.02)$ & 0.251 \\
Sex (ref.: male) & $1.01(0.46-2.21)$ & 0.985 \\
Underlying disease (ref.: CF) & & \\
$\quad$ COPD & $0.64(0.19-2.15)$ & 0.472 \\
ILD & $0.93(0.27-3.21)$ & 0.903 \\
Others & $1.87(0.49-7.13)$ & 0.362 \\
Severity of CLAD, FEV1/FEV1 & $0.97(0.94-0.99)$ & $\mathbf{0 . 0 1 7}$ \\
Time to CLAD years & $0.94(0.78-1.13)$ & 0.518 \\
Anterolateral thoracotomy (ref.: clamshell thoracotomy) & $1.39(0.54-3.55)$ & 0.494 \\
Azithromycin (ref.: no azithromycin) & $0.20(0.07-0.56)$ & $\mathbf{0 . 0 0 2}$ \\
Cyclosporine A (ref.: tacrolimus) & $2.70(0.97-7.57)$ & 0.058 \\
Year of transplantation & $1.18(1.06-1.31)$ & $\mathbf{0 . 0 0 3}$ \\
\hline
\end{tabular}

Data are presented as hazard ratios (HR) with $95 \%$ confidence intervals and p-values from two multiple regression models with outcome post-chronic lung allograft dysfunction (CLAD) survival. non-R/AT-CLAD: neither R- nor AT-CLAD; CF: cystic fibrosis; COPD: chronic obstructive lung disease; ILD: interstitial lung disease; FEV1: forced expiratory volume in $1 \mathrm{~s}$. Bold type indicates significant values.

\section{TABLE 3 Multiple Cox regression models assessing the effect of total lung capacity (TLC)/} $\mathrm{TLC}_{\text {baseline }}$ and residual volume (RV)/TLC on survival

\begin{tabular}{|c|c|c|}
\hline Covariate & $\operatorname{HR}(95 \% \mathrm{CI})$ & p-value \\
\hline TLC/TLC $C_{\text {baseline }}$ & & $0.044^{\#}, 0.100^{\# \#}$ \\
\hline Age & $1.00(0.97-1.03)$ & 0.980 \\
\hline Sex (ref.: male) & $0.71(0.38-1.29)$ & 0.260 \\
\hline \multicolumn{3}{|c|}{ Underlying disease (ref.: CF) } \\
\hline COPD & $1.29(0.53-3.13)$ & 0.570 \\
\hline ILD & $0.52(0.20-1.37)$ & 0.190 \\
\hline Others & $0.65(0.26-1.66)$ & 0.370 \\
\hline RV/TLC & & $<0.0001^{\#}, 0.181^{\# \#}$ \\
\hline Age & $0.98(0.96-1.01)$ & 0.308 \\
\hline Sex (ref.: male) & $0.66(0.36-1.21)$ & 0.177 \\
\hline \multicolumn{3}{|c|}{ Underlying disease (ref.: CF) } \\
\hline COPD & $1.30(0.52-3.24)$ & 0.577 \\
\hline ILD & $0.57(0.22-1.49)$ & 0.255 \\
\hline Others & $0.94(0.35-2.51)$ & 0.895 \\
\hline
\end{tabular}

Data are presented as hazard ratios (HR) with $95 \%$ confidence intervals and p-values from multiple regression models with outcome post-chronic lung allograft dysfunction survival. The estimated effect is nonlinear and can only be described via a graph (figure 3). CF: cystic fibrosis; COPD: chronic obstructive lung disease; ILD: interstitial lung disease. ${ }^{\#}$ : $p$-value for a linear trend. ${ }^{\# \#}$ : $p$-value for nonlinearity. 

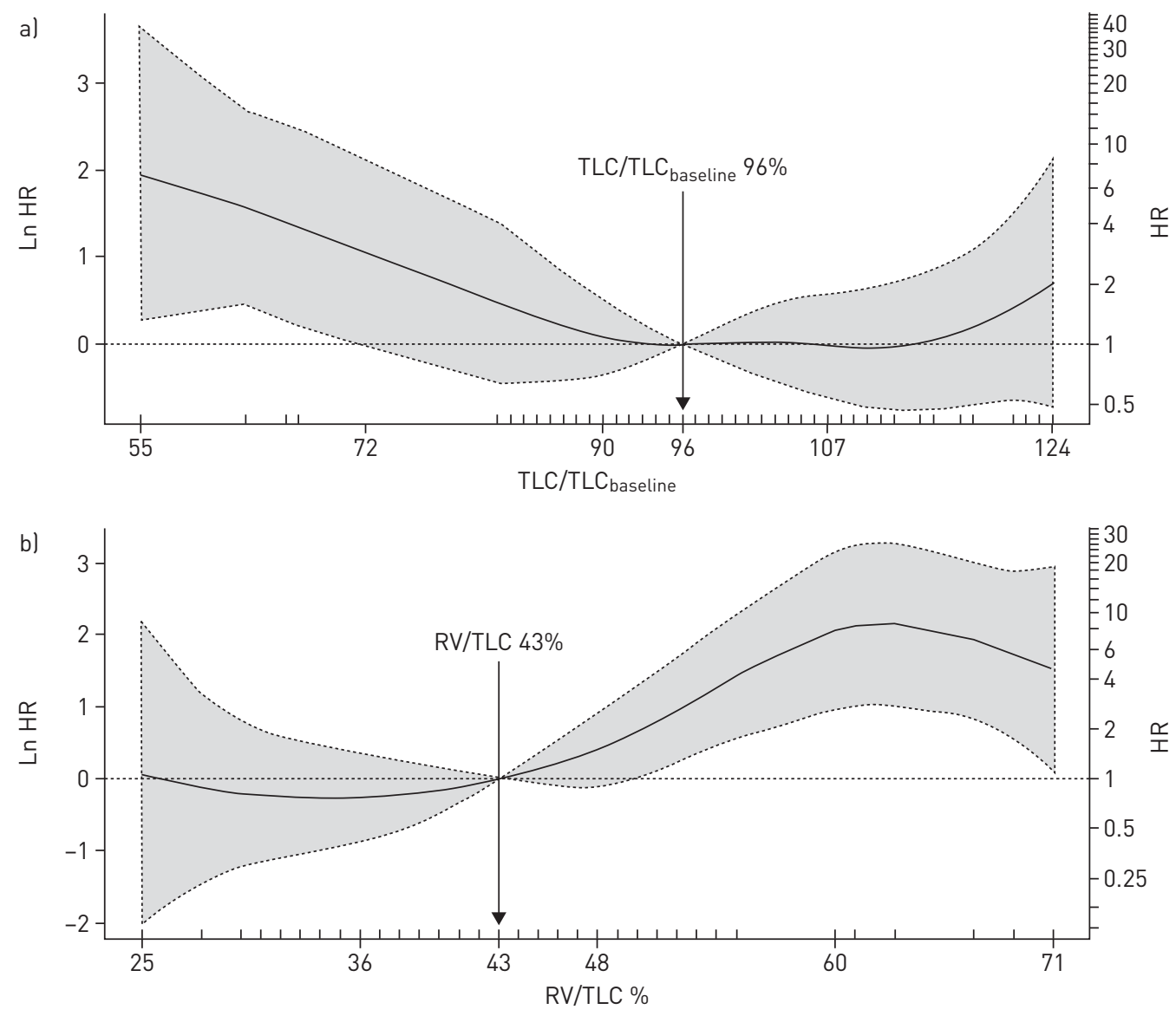

FIGURE 3 Effects of total lung capacity (TLC)/TLC baseline (a) and residual volume (RV)/TLC (b) represented by smooth, nonlinear functions. The solid curve is the estimated (log) hazard ratio (HR) as a function of $\mathrm{TLC} / \mathrm{TLC}_{\text {baseline. }}$. The hazard ratios are interpreted with respect to a patient with a mean $\mathrm{TLC} / \mathrm{TLC}_{\text {baseline }}$ of $96 \%$ (a) and mean RV/TLC of $43 \%$ (b). The grey area represents the $95 \%$ pointwise confidence intervals. Data are derived from all $(\mathrm{n}=94)$ patients.

R-CLAD or AT-CLAD only (supplementary figure S4). Radiographic findings and a representative chest CT scan of a patient with a combined phenotype (supplementary figure S5) are provided in the supplementary material.

TABLE 4 Radiographic findings on chest computed tomography scan at the time of chronic lung allograft dysfunction (CLAD) onset in patients with restrictive CLAD (R-CLAD) and CLAD with air trapping (AT-CLAD)

\begin{tabular}{lcc} 
& R-CLAD & AT-CLAD \\
\hline Subjects $\mathbf{n}$ & 20 & 21 \\
Centrilobular micronodules/tree in bud & $2(10.0)$ & $11(52.4)$ \\
Bronchiectasis/bronchial wall thickening & $8(40.0)$ & $13(61.9)$ \\
Interstitial changes & $19(95.0)$ & $10(47.6)$ \\
$\quad$ Predominant upper lung & $7(36.8)$ & $0(0.0)$ \\
Predominant middle lung & $1(5.3)$ & $0(0.0)$ \\
Predominant lower lung & $8(42.1)$ & $9(42.9)$ \\
Diffuse distribution & $3(15.8)$ & $1(4.8)$ \\
Ground glass opacities & $10(50.0)$ & $7(33.3)$ \\
Pleural thickening & $8(40.0)$ & $2(9.5)$ \\
Emphysema & $1(5.0)$ & $5(23.8)$ \\
\hline
\end{tabular}

Data are presented as $\mathrm{n}(\%)$, unless otherwise stated. ${ }^{\#}$ : consolidative opacities, septal thickening or reticulation. 


\section{Discussion}

We have investigated the utility of longitudinal lung volume measurements in the surveillance of lung transplant recipients. We have demonstrated that comprehensive pulmonary function tests permit the identification of physiological phenotypes of CLAD and, moreover, that pulmonary restriction and air trapping in lung function tests are associated with worse outcomes and thus warrant special consideration.

Fibrotic changes of the allograft as signs of chronic rejection were recognised several years ago, and the corresponding clinical syndrome was identified recently [2,3]. RAS is a condition associated with parenchymal infiltrates that is characterised by a more progressive course of CLAD. However, the diagnostic criteria for RAS are complex and are based on pulmonary function tests, imaging studies and histopathological characteristics. These have to be interpreted in the context of the clinical course [4]. SATO et al. [3] described a good agreement of a loss of TLC $<90 \%$ of baseline with the clinical diagnosis of RAS, whereas SuHLING et al. [21] recently suggested a cut-off of TLC $\angle 80 \%$ as the definition for restrictive CLAD. In our analysis, the high diagnostic accuracy of TLC $<90 \%$ (i.e. R-CLAD) was confirmed. It was associated with a high sensitivity (95\%) and specificity (97\%) for the clinical diagnosis of RAS.

Patients with R-CLAD were more likely to have COPD and less likely to have ILD as their pre-transplant diagnosis than patients with neither R- nor AT-CLAD. This association is in contrast to previous reports $[3,6,22,23]$ and should be investigated further in larger cohorts of patients with CLAD.

In patients with CLAD, a loss of TLC was identified as a prognostic marker for worse outcome in the multiple regression analysis. A TLC of less than $90 \%$ of the baseline value at CLAD onset was associated with worse survival after CLAD onset, irrespective of time to CLAD and severity of CLAD. The risk for unfavourable outcome increased linearly with decreasing TLC. A loss of TLC early after transplantation seemed to be particularly unfavourable, whereas a loss of TLC in the later period after transplantation might be less harmful. This further reflects the heterogeneous nature of RAS, as suggested previously [4]. An early onset and rapid progressive course might warrant a more aggressive approach than a late onset, chronic course of disease. In particular, recent successful reports of antifibrotic treatment in patients with RAS [24, 25] suggest the potential clinical value of early identification of lung transplant recipients with R-CLAD.

Unfortunately, serial TLC measurements are not routinely performed in many transplant centres. Recently, a loss of FVC at CLAD onset was identified as a prognostic and diagnostic marker for restrictive lung function decline $[6,7]$. Whether serial TLC measurements are superior to spirometric assessment of FVC needs to be investigated in large cohorts of patients with CLAD. In CT scans, R-CLAD was characterised by interstitial changes, with upper as well as lower lung predominant phenotypes.

By assessing the ratio of the volume remaining in the lung at the end of exhalation (RV) to the maximum volume capable of being inhaled (TLC), the degree of air trapping can be determined. Lung transplant recipients with air trapping at CLAD onset were at particular risk for poor outcome. In these patients CLAD was more severe at onset. However, the ratio of FEV1 to FVC was similar in patients with AT-CLAD and patients with non-R/AT-CLAD. Additionally, in the multiple regression analysis, air trapping was strongly associated with worse survival, while controlling for severity of CLAD at onset. Taken together, these data permitted us to identify a new and distinct physiological phenotype of CLAD by lung volume measurement, which cannot be attributed solely to the severity of CLAD at onset.

AT-CLAD occurred earlier than non-R/AT-CLAD. However, in contrast to R-CLAD, the detrimental effects of AT-CLAD on survival seemed to be independent of the time of onset. In the context of the cut-off chosen, AT-CLAD was as frequent as R-CLAD, but patients with AT-CLAD had lower median survival rates and survival times upon CLAD onset than patients with R-CLAD. Patients with AT-CLAD presented with various findings in CT scans, but signs of bronchiolitis, bronchiectasis and interstitial changes were predominant. Similar to our observations, air trapping is commonly seen with bronchiectasis and ILDs in imaging studies [26]. The value of CT scans in in- and expiration to assess the degree of air trapping in patients with BOS has been reported previously [27-29] and would strengthen the concept of this new phenotype. Unfortunately, these were not available throughout this entire study period.

The cut-off at $50 \%$ for AT-CLAD was chosen based on a post hoc risk and approximates to the degree of air trapping of a large cohort of patients with obstructive lung diseases [30]. However, the cut-off needs to be confirmed in different cohorts of patients with CLAD and correlated to other clinical characteristics. Irrespective of a particular threshold, RV/TLC as a continuous variable was strongly associated with survival. The risk for a higher hazard was noticed at $45 \%$ and increased linearly.

Air trapping was assessed both by a single measure of RV/TLC at CLAD onset and by a change over time. Interestingly, the single measurement was associated with a strong prognostic value, whereas an increase of RV/TLC over the post-transplant period was less specific. This suggests that the presence of air trapping might be more relevant than dynamic changes over time. 
Air trapping refers to the retention of inspired air during expiration, either as a result of complete or partial airway closure. It has been associated with different diseases [30-33] and is commonly seen in combination with various airway and parenchymal changes [26]. The reason for worse outcome in patients with CLAD and air trapping needs to be studied in further detail. A clinical consequence of air trapping is pulmonary hyperinflation, which has several detrimental effects, including impairment of respiratory muscle function and gas exchange, increased work in breathing and dyspnoea, and impaired exercise performance [34]. Similar effects might be responsible for early death after CLAD onset in lung transplant recipients, but this requires further exploration. However, an increase of RV/TLC is less specific for assessing pulmonary hyperinflation than measurements of functional residual capacity, and may also be present when RV remains static or increases out of proportion to the fall in TLC.

In this context, $6.4 \%$ of patients fulfilled the criteria for both R- and AT-CLAD, indicating a combined phenotype of CLAD. It has previously been observed that RAS and BOS might occur consecutively or even simultaneously $[3,4]$. In our cohort, a combined phenotype was associated with a worse survival compared to non-R/AT-CLAD. However, it was no different to R-CLAD and AT-CLAD only, respectively. The number of patients with the combined phenotype was rather small. Hence, to reliably and thoroughly characterise this phenotype, larger cohorts are needed. Lung volume measurement might be useful to identify this specific population of patients for further characterisation.

The results of our study should be interpreted in view of its limitations. First, the study is a single-centre study with a mid-European lung transplant cohort and might not apply entirely to other populations. Secondly, the study is retrospective, spanning a period of over two decades. Even though various variables, which changed over the study period, were considered in our analysis, the changing treatment of lung transplant recipients over time might have impacted the course of CLAD. Diaphragmatic palsy was not systematically assessed in our study and might influence lung volume measurements. However, we provide data from one of the largest cohorts of patients with CLAD reported so far. Because all patients are followed up in our centre, irrespective of time after transplantation, and only bilateral lung transplant recipients were included, our cohort is well characterised. Furthermore, we had access to a substantial and detailed dataset, derived from over 9000 pulmonary function tests.

In conclusion, lung volume measurements in the surveillance of lung transplant recipients has permitted us to identify patient populations with specific physiological phenotypes of CLAD that are at risk for worse outcomes. This offers the potential for early, and targeted, clinical intervention.

\section{References}

1 Verleden GM, Raghu G, Meyer KC, et al. A new classification system for chronic lung allograft dysfunction. $J$ Heart Lung Transplant 2014; 33: 127-133.

2 Konen E, Weisbrod GL, Pakhale S, et al. Fibrosis of the upper lobes: a newly identified late-onset complication after lung transplantation? AJR Am J Roentgenol 2003; 181: 1539-1543.

3 Sato $\mathrm{M}$, Waddell TK, Wagnetz $\mathrm{U}$, et al. Restrictive allograft syndrome (RAS): a novel form of chronic lung allograft dysfunction. J Heart Lung Transplant 2011; 30: 735-742.

4 Verleden SE, Ruttens D, Vandermeulen E, et al. Restrictive chronic lung allograft dysfunction: where are we now? $J$ Heart Lung Transplant 2015; 34: 625-630.

5 Kapila A, Baz MA, Valentine VG, et al. Reliability of diagnostic criteria for bronchiolitis obliterans syndrome after lung transplantation: a survey. J Heart Lung Transplant 2015; 34: 65-74.

6 Todd JL, Jain R, Pavlisko EN, et al. Impact of forced vital capacity loss on survival after the onset of chronic lung allograft dysfunction. Am J Respir Crit Care Med 2014; 189: 159-166.

7 Belloli EA, Wang X, Murray S, et al. Longitudinal forced vital capacity monitoring as a prognostic adjunct after lung transplantation. Am J Respir Crit Care Med 2015; 192: 209-218.

8 Verleden GM, Vos R, Verleden SE, et al. Survival determinants in lung transplant patients with chronic allograft dysfunction. Transplantation 2011; 92: 703-708.

9 Casanova C, Cote C, de Torres JP, et al. Inspiratory-to-total lung capacity ratio predicts mortality in patients with chronic obstructive pulmonary disease. Am J Respir Crit Care Med 2005; 171: 591-597.

10 Budweiser S, Harlacher M, Pfeifer M, et al. Co-morbidities and hyperinflation are independent risk factors of all-cause mortality in very severe COPD. COPD 2014; $11: 388-400$.

11 Lahaije AJMC, van Helvoort HAC, Dekhuijzen PNR, et al. Resting and ADL-induced dynamic hyperinflation explain physical inactivity in COPD better than FEV1. Respir Med 2013; 107: 834-840.

12 Kneidinger N, Holzborn J, Czerner S, et al. Evaluation of short-term outcome after lung transplantation in the lung allocation score era. Thorac Cardiovasc Surg 2015; 63: 693-698.

13 Estenne M, Maurer JR, Boehler A, et al. Bronchiolitis obliterans syndrome 2001: an update of the diagnostic criteria. J Heart Lung Transplant 2002; 21: 297-310.

14 Meyer KC, Raghu G, Verleden GM, et al. An international ISHLT/ATS/ERS clinical practice guideline: diagnosis and management of bronchiolitis obliterans syndrome. Eur Respir J 2014; 44: 1479-1503.

15 Sato M, Hwang DM, Waddell TK, et al. Progression pattern of restrictive allograft syndrome after lung transplantation. J Heart Lung Transplant 2013; 32: 23-30.

16 Copeland CA, Snyder LD, Zaas DW, et al. Survival after bronchiolitis obliterans syndrome among bilateral lung transplant recipients. Am J Respir Crit Care Med 2010; 182: 784-789. 
17 Harrington DP, Fleming TR. A class of rank test procedures for censored survival data. Biometrika 1982; 69: 553-566.

18 Kaplan EL, Meier P. Nonparametric estimation from incomplete observations. J Am Stat Assoc 1958; 53: 457-481.

19 Cox DR. Regression models and life tables. J R Stat Soc 1972; 34: 187-220.

20 R Core Team. R: a language and environment for statistical computing. Vienna, R Foundation for Statistical Computing 2013.

21 Suhling $\mathrm{H}$, Dettmer S, Greer $\mathrm{M}$, et al. Phenotyping chronic lung allograft dysfunction using body plethysmography and computer tomography. Am J Transplant 2016; 16: 3163-3170.

22 Woodrow JP, Shlobin OA, Barnett SD, et al. Comparison of bronchiolitis obliterans syndrome to other forms of chronic lung allograft dysfunction after lung transplantation. J Heart Lung Transplant 2010; 29: 1159-1164.

23 Verleden SE, Ruttens D, Vandermeulen E, et al. Bronchiolitis obliterans syndrome and restrictive allograft syndrome: do risk factors differ? Transplantation 2013; 95: 1167-1172.

24 Vos R, Verleden SE, Ruttens D, et al. Pirfenidone: a potential new therapy for restrictive allograft syndrome? Am J Transplant 2013; 13: 3035-3040.

25 Suhling H, Bollmann B, Gottlieb J. Nintedanib in restrictive chronic lung allograft dysfunction after lung transplantation. J Heart Lung Transplant 2016; 35: 939-940.

26 Miller WT Jr, Chatzkel J, Hewitt MG. Expiratory air trapping on thoracic computed tomography. A diagnostic subclassification. Ann Am Thorac Soc 2014; 11: 874-881.

27 Leung AN, Fisher K, Valentine V, et al. Bronchiolitis obliterans after lung transplantation: detection using expiratory HRCT. Chest 1998; 113: 365-370.

28 Lee ES, Gotway MB, Reddy GP, et al. Early bronchiolitis obliterans following lung transplantation: accuracy of expiratory thin-section CT for diagnosis. Radiology 2000; 216: 472-477.

29 Siegel MJ, Bhalla S, Gutierrez FR, et al. Post-lung transplantation bronchiolitis obliterans syndrome: usefulness of expiratory thin-section CT for diagnosis. Radiology 2001; 220: 455-462.

30 Dykstra BJ, Scanlon PD, Kester MM, et al. Lung volumes in 4,774 patients with obstructive lung disease. Chest 1999; 115: 68-74.

31 Hartley RA, Barker BL, Newby C, et al. Relationship between lung function and quantitative computed tomographic parameters of airway remodeling, air trapping, and emphysema in patients with asthma and chronic obstructive pulmonary disease: a single-center study. J Allergy Clin Immunol 2016; 137: 1413-1422.

32 Hansell DM, Wells AU, Padley SP, et al. Hypersensitivity pneumonitis: correlation of individual CT patterns with functional abnormalities. Radiology 1996; 199: 123-128.

33 Davies CW, Tasker AD, Padley SP, et al. Air trapping in sarcoidosis on computed tomography: correlation with lung function. Clin Radiol 2000; 55: 217-221.

34 American Thoracic Society/European Respiratory Society. ATS/ERS statement on respiratory muscle testing. Am J Respir Crit Care Med 2002; 166: 518-624. 